\title{
Equivalent temperature of nonlinear-optical crystals interacting with laser radiation
}

\section{O. A. Ryabushkin}

\section{V. Myasnikov}

\section{A. V. Konyashkin}

\section{A. Tyrtyshnyy}

\author{
NTO “IRE-Polus”, Vvedensky Sq.1, Fryazino, Moscow region, 141190, Russia \\ Moscow Institute of Physics and Technology, Institutskii per. 9, Dolgoprudny, Moscow region, 141700, \\ Russia \\ Kotelnikov Institute of Radio-engineering and Electronics of RAS, Vvedensky Sq.1, Fryazino, Moscow \\ region, 141190, Russia \\ NTO “IRE-Polus”, Vvedensky Sq.1, Fryazino, Moscow region, 141190, Russia \\ Moscow Institute of Physics and Technology, Institutskii per. 9, Dolgoprudny, Moscow region, 141700, \\ Russia \\ Kotelnikov Institute of Radio-engineering and Electronics of RAS, Vvedensky Sq.1, Fryazino, Moscow \\ region, 141190, Russia \\ NTO “IRE-Polus”, Vvedensky Sq.1, Fryazino, Moscow region, 141190, Russia \\ Moscow Institute of Physics and Technology, Institutskii per. 9, Dolgoprudny, Moscow region, 141700, \\ Russia \\ Kotelnikov Institute of Radio-engineering and Electronics of RAS, Vvedensky Sq.1, Fryazino, Moscow \\ region, 141190, Russia \\ NTO “IRE-Polus”, Vvedensky Sq.1, Fryazino, Moscow region, 141190, Russia \\ Moscow Institute of Physics and Technology, Institutskii per. 9, Dolgoprudny, Moscow region, 141700, \\ Russia \\ Kotelnikov Institute of Radio-engineering and Electronics of RAS, Vvedensky Sq.1, Fryazino, Moscow \\ region, 141190, Russia
}

Temperature calibrated piezoelectric resonances of internal acoustic vibration modes of a nonlinear-optical crystal during its heating by high-power laser radiation are used for noncontact measurements of both the non-uniform temperature distribution in the crystal volume and in the surrounding air. A novel notion of equivalent temperature of a crystal heated by laser radiation is introduced in laser physics. The true non-uniform crystal thermodynamic temperature at a given laser power is substituted by the measured equivalent crystal temperature, which is constant at that laser power. Using appropriate laser heating model the measured value of the equivalent crystal temperature allows one to calculate the unknown linear and nonlinear optical absorption coefficients as well as the heat transfer coefficient of the crystal with the surrounding air. [DOI: 10.2971/jeos.2011.11032]

Keywords: piezoelectric resonance, nonlinear-optical crystals, optical absorption, heat transfer coefficient

\section{INTRODUCTION}

Frequency conversion efficiency of laser radiation in nonlinear-optical crystals is governed by a phase matching condition [1]. One of the essential requirements for providing the phase matching condition is crystal temperature control. Until now there are no precision methods developed for crystal temperature control for high-power laser frequency conversion applications. The main reason for that is lack of a simple noncontact technique of the real-time crystal temperature determination during nonlinear-optical conversion process. That can lead to conversion efficiency decrease as well as to formation of defects (gray track) and even further to irreversible optical damage of the crystal [2]-[5]. The reason for that is primarily that during laser frequency conversion crystal temperature nonlinearly increases with pump laser power. The crystal temperature rise is caused not only by the change of the optical pump absorption coefficient but also, what is more essential, by the uncontrollable increase of the converted radiation absorption. In conventional contact methods the crystal surrounding air temperature is measured [6]. The required precision cannot be achieved in such techniques due to a high temperature gradient near the heated crystal surface. Furthermore, during nonlinear-optical conversion process the heat exchange conditions of the crystal-air boundaries may also vary. The main goal of our present research is the development of the precise method for crystal temperature control during laser heating. The method proposed here is based on the measurement of electrical impedance spectra of nonlinear-optical crystals in the radio-frequency $(\mathrm{RF})$ domain. After conducting preliminary experiments it became evident that a theoretical model is needed for the laser heating process description. This model is based on direct independent measurements of the sur- 
rounding air temperature and the crystal temperature during its interaction with laser radiation of laser power $P_{i n}$. Here the nonlinear-optical crystal temperature is determined directly from the measured piezoelectric resonance frequency $R f_{n}$ of any $(n)$ internal vibration mode. In turn, the surrounding air temperature is measured using additional piezoelectric thermal resonators, placed near the nonlinear-optical crystal. The nonlinear-optical crystal temperature for the incident laser power $P_{i n}$ is determined by measuring the frequency shift $\Delta R f_{n}\left(P_{i n}\right)$ of the temperature $\theta$ calibrated piezoelectric resonance frequency $R f_{n}(\theta)$. Relying on the experimental measurements of the $R f_{n}(\theta), \Delta R f_{n}\left(P_{\text {in }}\right)$ dependencies together with theoretical modeling of crystal non-uniform heating we were able to introduce a novel notion in laser physics - "the equivalent crystal heating temperature $\Delta \Theta^{e q}\left(P_{\text {in }}\right)^{\prime \prime}$. This gave us a possibility to substitute the non-uniform crystal heating temperature $\Delta \theta_{c r}\left(x, y, z, P_{\text {in }}\right)$, which can only be calculated for a given laser power $P_{i n}^{f}$, by the measured value $\Delta \Theta^{e q}\left(P_{i n}^{f}\right)$, which is constant for that $P_{i n}$. The equivalent crystal temperature is determined by the following simple expression: $\Theta^{e q}\left(P_{i n}^{f}\right)=\theta_{a}+\Delta \Theta^{e q}\left(P_{i n}^{f}\right)$. Here $\theta_{a}$ is the surrounding air temperature that is measured before the laser is turned on [7].

It is well known that nonlinear-optical crystals with non-zero second order susceptibility posses piezoelectric properties. Due to the inverse piezoelectric effect the external ac electric field applied to the crystal can excite its acoustical vibrations. Piezoelectric resonance is observed when an electric field frequency from an RF generator $f g$ coincides with one of the internal acoustical vibration mode frequencies $\left(R f_{n}=f g\right)$. The piezoelectric resonance frequencies $R f_{n}$ depend not only on internal factors (crystal dimensions and shape; symmetry of the electric, elastic and piezoelectric tensors) but also on external factors (surrounding air pressure and humidity; direction and inhomogeneity of the external AC electric field etc.). Certain piezoelectric resonances are strongly sensitive to the crystal temperature change. There are versatile experimental techniques for registration of piezoelectric resonances in the wide frequency range of $10^{-3}-10^{10} \mathrm{~Hz}$. We have employed these techniques in an experimental setup, which we developed for measurement of piezoelectric resonances of nonlinear-optical crystals during their interaction with single-mode laser radiation. The temperature control of both the crystal and the surrounding air was realized with $10 \mathrm{mK}$ accuracy [8]. We have observed that piezoelectric resonance frequencies of nonlinear-optical crystals $\left(\mathrm{LiNbO}_{3}\right.$, $\mathrm{KH}_{2} \mathrm{PO}_{4}, \mathrm{KTiOPO}_{4}, \mathrm{LiB}_{3} \mathrm{O}_{5}$ and many others) widely used for laser frequency conversion are highly sensitive even to lowpower radiation influence. It should be mentioned that in the $90^{\text {th }}$ of the previous century [9] and in the beginning of our century [10] there were several attempts made to exploit the piezoelectric resonance sensitivity to weak laser radiation for determination of the low optical absorption coefficients of nonlinear-optical crystals. Unfortunately the hasty conclusions of the second paper [10] have limited the application of the proposed method to the registration of the nonlinearoptical crystal temperature change caused by laser radiation heating of extremely low power (below $30 \mathrm{~mW}$ ). Our experiments show that temperature measurement of the nonlinearoptical crystals exploiting acousto-resonant technique is not limited by laser powers of $\mathrm{mW}$ level. Application of our method is limited only by the laser power values that cause optical damage of the crystal. Thus laser powers can reach $\mathrm{kW}$ level in the CW regime of operation [11].

\section{EXPERIMENTAL}

\subsection{Experimental Setup}

The experimental study of the nonlinear-optical crystals $\mathrm{SiO}_{2}$, $\mathrm{KTiOPO}_{4}, \mathrm{KH}_{2} \mathrm{PO}_{4}, \mathrm{LiNbO}_{3}, \mathrm{LiB}_{3} \mathrm{O}_{5}$ was performed using an automated experimental setup shown in Figure 1(a).

Here we give only a short description of the experimental setup. More detailed description can be found elsewhere [8]. The nonlinear-optical crystal (sample crystal) was placed in the center of the capacitor formed by two metal plates. The distance between the capacitor plates was carefully chosen to meet two contradictory requirements. The first is that the RF electric field inside the capacitor in the vicinity of the sam-

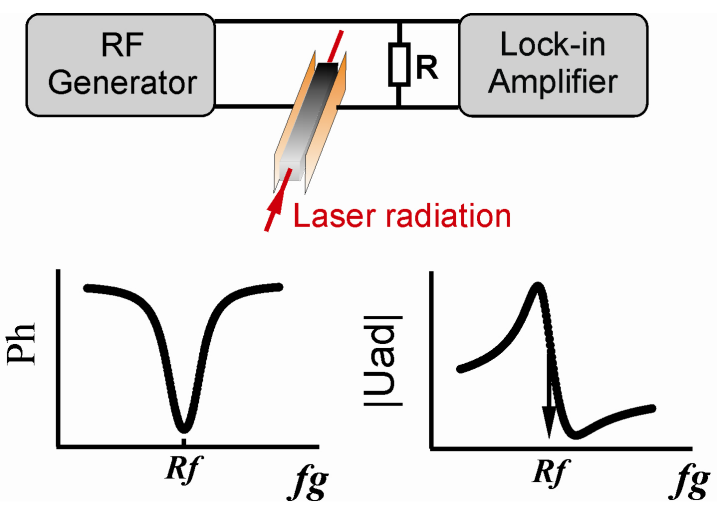

FIG. 1a Simplified block scheme of the experimental setup and typical dependencies of the voltage phase $\mathrm{Ph}$ and absolute voltage value $|\operatorname{Uad}(f g)|$, measured on the load resistor $\mathrm{R}$, on frequency in the vicinity of the crystal piezoelectric resonance.

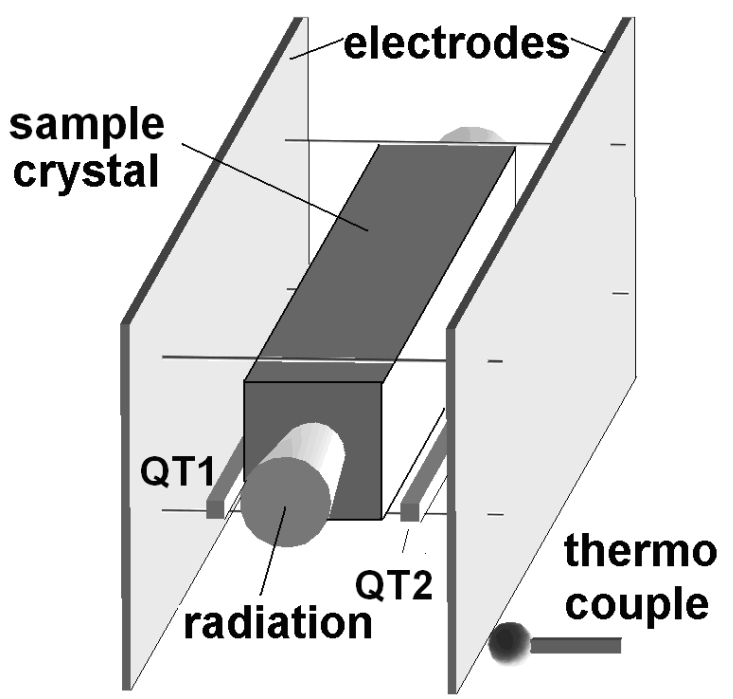

FIG. 1b Crystal arrangement inside the capacitor: sample crystal, thermo resonator crystals QT1 and QT2. 


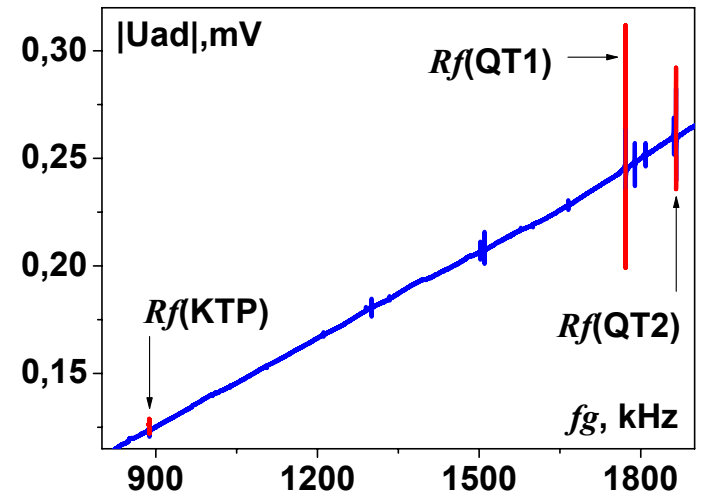

FIG. 2 Dependence of the absolute voltage value $|\operatorname{Uad}(f g)|$ on frequency measured for the KTP together with QT1 and QT2 crystals.

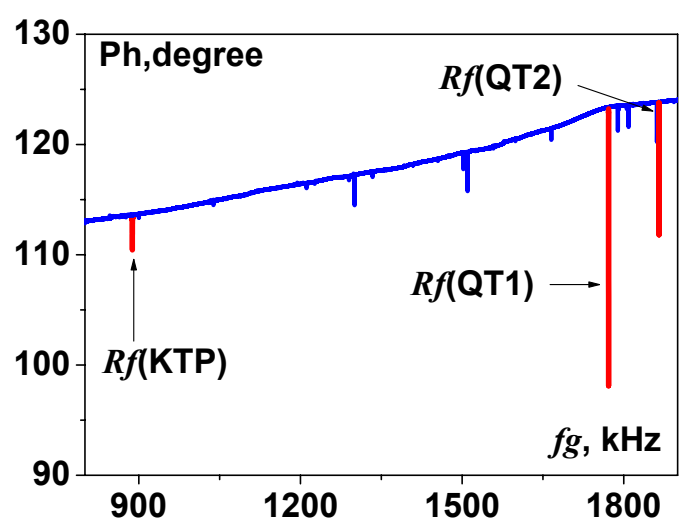

FIG. 3 Dependence of the voltage phase Ph on frequency measured for the KTP together with $\mathrm{QT} 1$ and $\mathrm{QT} 2$ crystals.

ple should be as uniform as possible. On the other hand, the distance between the electrodes should be large enough in order to reduce the influence of the electrodes heating caused by the scattered radiation from the crystal. Here the distance between the capacitor electrodes was set $10 \mathrm{~mm}$. In particular, to our opinion, uncontrollable heating by the scattered radiation of the electrodes deposited on the $\mathrm{LiNbO}_{3}$ facets was the main reason that drew the authors of paper [10] to conclusion that piezoelectric resonance cannot be used for the precise temperature measurements of the nonlinear-optical crystals interacting with high-power laser radiation. In order to control temperature of the electrodes as well as the air temperature inside the capacitor we placed two additional thermo resonators QT1 and QT2 (see Figure 1(b)) made of quartz crystals with a small cross section into the capacitor. The piezoelectric resonances of the sample crystal and two quartz crystals were observed in the overall impedance spectrum of the capacitor, connected in series with an RF generator and a small load resistor $\mathrm{R}=47 \mathrm{Ohm}$. The voltage on the load resistor $\mathrm{R}$ was measured by a lock-in amplifier. The voltage amplitude of the $\mathrm{RF}$ generator was kept at $1 \mathrm{~V}$ for all experiments. The sample crystal was heated by a CW single-mode linearly polarized ytterbium fiber laser manufactured by NTO "IRE-Polus". The highest output laser power was equal to $70 \mathrm{~W}$, the laser beam diameter equaled to $1.5 \mathrm{~mm}$ (FWHM). Here we present the results of the study of the nonlinear-optical crystal $\mathrm{KTiOPO}_{4}$ (KTP). The crystal had a shape of a rectangular parallelepiped with dimensions $L x=2.8 \mathrm{~mm}, L y=3.2 \mathrm{~mm}, L z=12 \mathrm{~mm}$. The front facets of the uncoated KTP were polished. Temperature of the air surrounding the KTP crystal can be determined at sites of the QT1 and QT2 crystals exploiting their piezoelectric thermal properties. The supplemental quartz crystals, used for the air temperature measurement, also were in the form of rectangular parallelepipeds with dimensions $L x \approx 1 \mathrm{~mm}$, $L y \approx 1 \mathrm{~mm}, L z \approx 11 \mathrm{~mm}$.

The $|\mathrm{Uad}|$ value is directly proportional to the admittance absolute value $|\mathrm{Yad}|$. The voltage phase $\mathrm{Ph}$ is the difference between the phase of the electrical current going through the capacitor and the generator voltage phase. The overall admittance Yad of the electrical chain is the sum of the capacitor admittance containing the KTP, QT1 and QT2 crystals and admittances of the connecting coaxial cables. Figure 2 and Figure 3 show the RF spectra for the absolute value $|\mathrm{Uad}|$ and the phase $\mathrm{Ph}$ of the voltage, measured on the load resistor R, for the KTP crystal and the QT1, QT2 thermo resonators at the air temperature $\theta_{a}=290.5 \mathrm{~K}$. Several resonance peaks with high Q-factors were clearly observed. Every resonance peak could be easily attributed to the corresponding crystal performing measurements with each crystal separately. Only one piezoelectric resonance in the selected frequency range $(800-1900 \mathrm{kHz})$, however was related to the KTP crystal: $R f(K T P)=887.980 \mathrm{kHz}(\mathrm{Phmin}=109.8 \mathrm{deg})$ others belong to QT1 and QT2. In addition to the value of $R f$, the shape of the spectral line of the absolute value $|\operatorname{Uad}(f g)|$ is also an important information source. The piezoelectric resonance line shape can be characterized by two parameters: amplitude of the absolute voltage value and the spectral line width. The resonance amplitude is determined as follows $\mathrm{Uad}=\mathrm{U}_{a d}^{\min }-\mathrm{U}_{a d}^{\max }$, where $\mathrm{U}_{a d}^{\min }$ and $\mathrm{U}_{a d}^{\max }$ are the minimum (at $R f \mathrm{~min}$ ) and the maximum (at $R f \max$ ) values of the $|\mathrm{Uad}(f g)|$ respectively. Resonance line width is calculated as $\mathrm{Wad}=R f \max -R f$ min. The values of these parameters measured for the selected crystals resonances (marked red in Figure 2, Figure 3) are presented in Table 1.

\begin{tabular}{|c|c|c|c|}
\hline Crystal & $R f_{n}, \mathrm{kHz}$ & $\mathrm{Wad}, \mathrm{Hz}$ & $\mathrm{Uad}, \mu \mathrm{V}$ \\
\hline KTP & 887.980 & 240 & 8.5 \\
\hline QT1 & 1771.812 & 45 & 113.3 \\
\hline QT2 & 1865.534 & 70 & 56.4 \\
\hline
\end{tabular}

TABLE 1 Piezoelectric resonance parameters for the KTP, QT1 and QT2 crystals at $\theta_{a}=290.5 \mathrm{~K}$.

\subsection{Piezoelectric resonance frequency temperature calibration}

The most sensitive to the temperature change piezoelectric resonances of the sample crystal (KTP) and the thermo resonators (QT1 and QT2) were calibrated with respect to temperature during uniform heating (without laser radiation) [8]. For all crystals studied dependence of the piezoelectric resonance frequencies shifts on temperature exhibit linear behavior in the temperature range of $290-370 \mathrm{~K}$ [8, 12]. For each crystal and each resonance corresponding piezoelectric resonance thermal coefficients were determined: $K^{\text {prt }}(\mathrm{KTP})$, $K^{\text {prt }}(\mathrm{QT} 1), K^{\text {prt }}(\mathrm{QT} 2)$. 


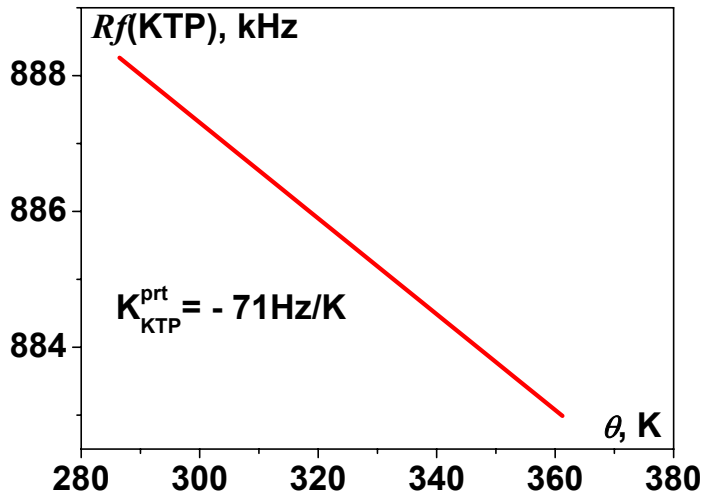

FIG. 4a Piezoelectric resonance frequency shift with temperature measured for the KTP crystal.

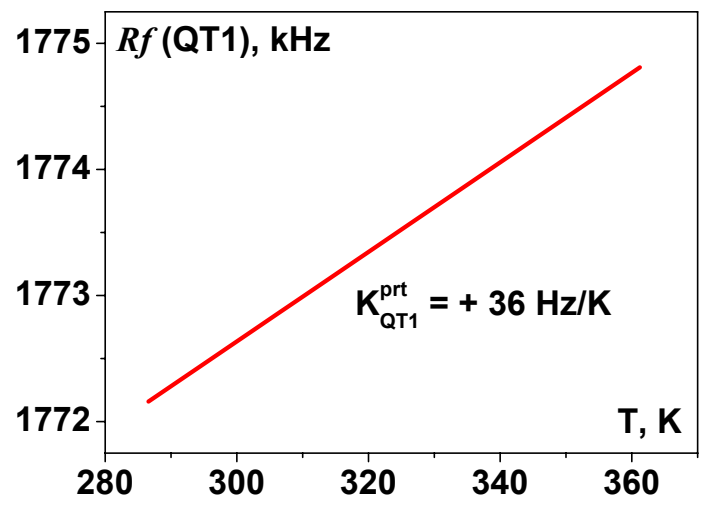

FIG. 5a Piezoelectric resonance frequency shift with temperature measured for the QT1 crystal.

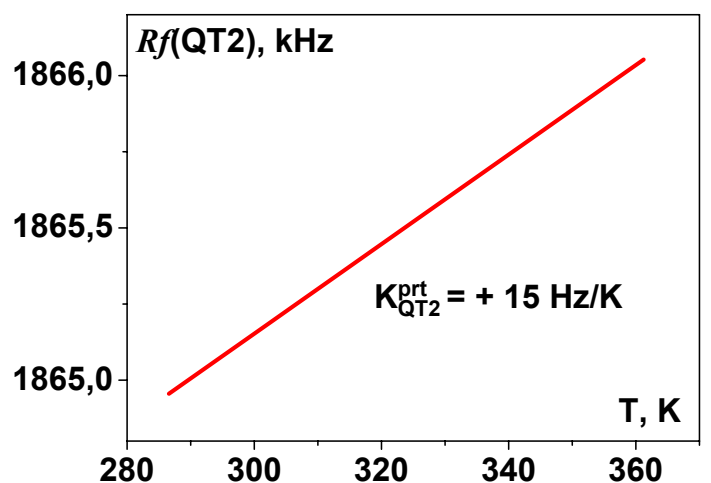

FIC. 6a Piezoelectric resonance frequency shift with temperature measured for the QT2 crystal.

Figure 4 shows typical temperature dependencies of the resonance frequency $R f(\theta)$ and absolute voltage value $|\mathrm{Uad}|$ measured for the KTP crystal piezoelectric resonance. Figure 5 and 6 present temperature dependencies of the resonance frequencies $R f(\mathrm{QT} 1)$ and $R f(\mathrm{QT} 2)$ and the voltage phases Ph obtained for the crystals QT1 and QT2 piezoelectric resonances. It can be seen that crystal heating was followed by the change in both the resonance line shape and the value of the piezoelectric resonance frequency. For the former we observed both the voltage absolute value $|\mathrm{Uad}|$ and phase Ph change in the vicinity of the resonance frequency.

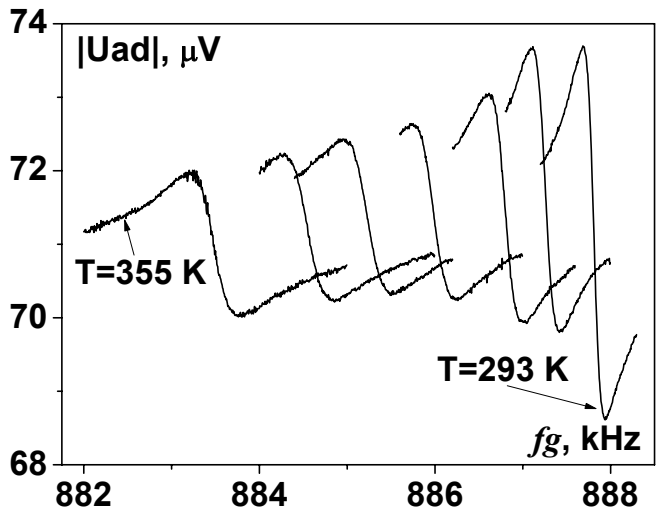

FIC. 4b Dependence of the absolute voltage value $|\operatorname{Uad}(f g)|$ on frequency in vicinity of the KTP piezoelectric resonance at different temperatures.

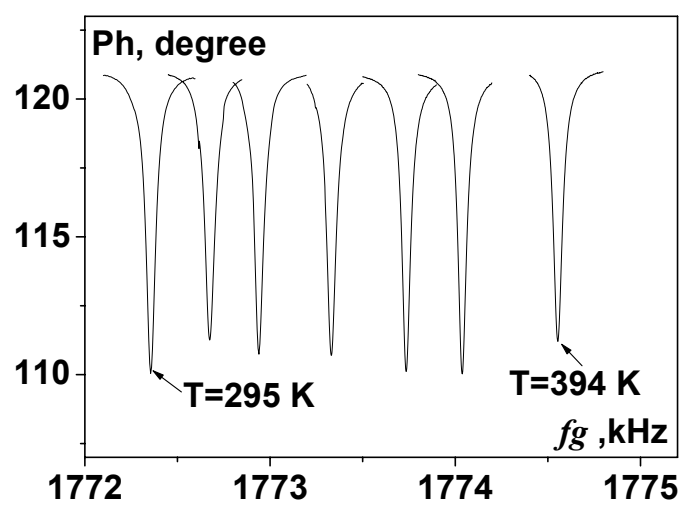

FIG. 5 b Dependence of the voltage phase Ph on frequency in vicinity of the QT1 piezoelectric resonance at different temperatures.

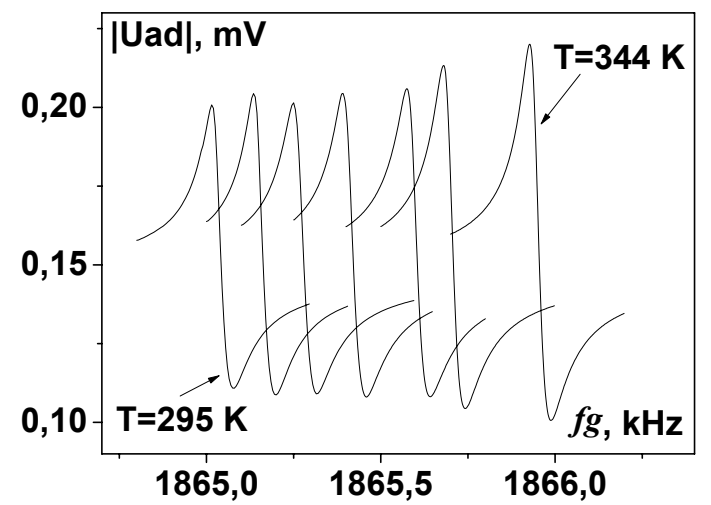

FIG. $6 \mathrm{~b}$ Dependence of the absolute voltage value $|\operatorname{Uad}(f g)|$ on frequency in vicinity of the QT2 piezoelectric resonance at different temperatures.

According to the experimental results the resonance amplitude Uad and its spectral line width Wad non-monotonically depend on temperature. The spectral line width of the KTP crystal resonance strongly increases with temperature. At the same time such behavior was not observed for the QT1 and QT2 crystals.

The piezoelectric resonance thermal coefficient of a certain mode, $K_{n}^{p r t}=d R f_{n}(\theta) / d \theta$ determines the resonance frequency shift with temperature during the uniform crystal 
temperature change. Based on the experimental data the piezoelectric resonance frequency $R f_{n}(\theta)$ linearly depends on temperature and therefore can be obtained using the following formula $[8,12]$ :

$$
R f_{n}(\theta)=R f_{n}\left(\theta_{a}\right)+K_{n}^{p r t}\left(\theta-\theta_{a}\right)
$$

Here frequency $R f_{n}\left(\theta_{a}\right)$ corresponds to the initial temperature $\theta_{a}$ of the air surrounding the crystal. The absolute value and the sign of the $K_{n}^{p r t}$ coefficients depend on the selected vibration mode of the crystal. We obtained the following values of $K_{n}^{p r t}$ for our KTP, QT1 and QT2 crystals resonances: $K^{p r t}(\mathrm{KTP})=-71 \mathrm{~Hz} / \mathrm{K}, K^{p r t}(\mathrm{QT} 1)=36 \mathrm{~Hz} / \mathrm{K}$ and $K^{p r t}(\mathrm{QT} 2)=15 \mathrm{~Hz} / \mathrm{K}$.

\subsection{Determination of the KTP crystal heating by laser radiation}

Under laser action, the sample crystal temperature can be determined by measuring the resonance frequency change $\Delta R f(\mathrm{KTP})$. Similarly, temperature of the air surrounding the sample crystal can be determined from $\Delta R f(\mathrm{QT1})$ and $\Delta R f(\mathrm{QT} 2)$. In these experiments we measured not only the incident laser power $P_{\text {in }}$, but also the output power $P_{\text {out }}$, which can be expressed as

$$
P_{\text {out }}=P_{\text {in }}-P_{r}-P_{a}-P_{s},
$$

where $P_{r}$ is the power of the light reflected from the crystal facets, $P_{a}$ and $P_{S}$ are the powers of the absorbed and scattered light by the crystal respectively. In Figure 7 the dependence of the output power on the incident power for the KTP crystal is presented. It should be noted that in all our experiments $P_{r}$ as well as $P_{s}$ were much larger than $P_{a}$. $P_{r}$ can be significantly reduced by applying AR coating to the crystal facets, while scattered power $P_{S}$ cannot be easily weakened and can heat elements surrounding the crystal. Crystal heating is determined only by $P_{a}$. Equation (2) can be rewritten as

$$
K_{\text {out }}+K_{r}+K_{s}+K_{a}=1,
$$

where

$$
K_{\text {out }}=P_{\text {out }} / P_{\text {in }}, K_{r}=P_{r} / P_{\text {in }}, K_{s}=P_{s} / P_{\text {in }}, K_{a}=P_{a} / P_{\text {in }} .
$$

$K_{\text {out }}$ characterizes the output power, $K_{r}$ represents the reflected power, $K_{s}$ corresponds to the scattered power, $K_{a}$ is related to the absorbed power. For our KTP crystal these coefficients were measured to be $K_{o u t}=0.82, K_{r}=0.15, K_{s}=0.03$. As it will be shown later, $K_{a} \approx 0.001$.

If laser power $P_{\text {in }}$ with intensity distribution $I_{\text {in }}(x, y)$ is launched into the crystal, resonance frequency $R f_{n}\left(P_{i n}\right)$, measured after thermal equilibrium is reached, can be expressed as a linear function of $P_{\text {in }}$ with a piezoelectric resonance optical coefficient

$$
K_{n}^{\text {pro }}=d R f_{n}\left(P_{\text {in }}\right) / d P .
$$

Piezoelectric resonance frequency shift with the incident laser power measured for the KTP crystal is shown in Figure 8. Dependencies of the QT1 and QT2 piezoelectric resonance frequencies on the KTP incident laser power are also shown in Figure 9 and Figure 10 respectively.

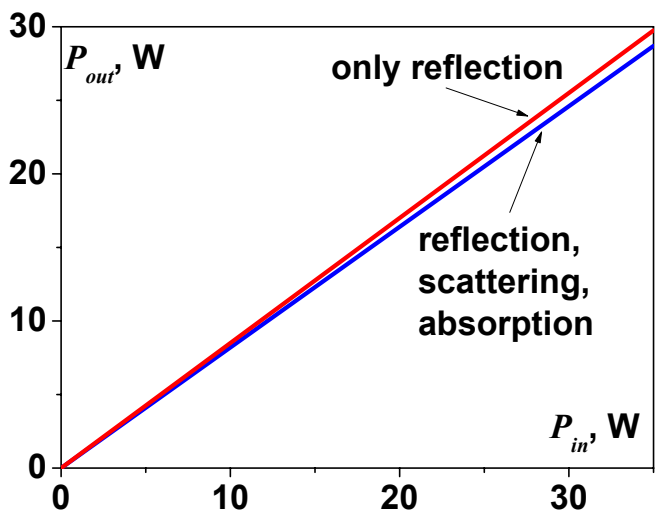

FIC. 7 Dependence of the output laser power $P_{\text {out }}$ on the incident laser power $P_{\text {in }}$ for the KTP crystal.

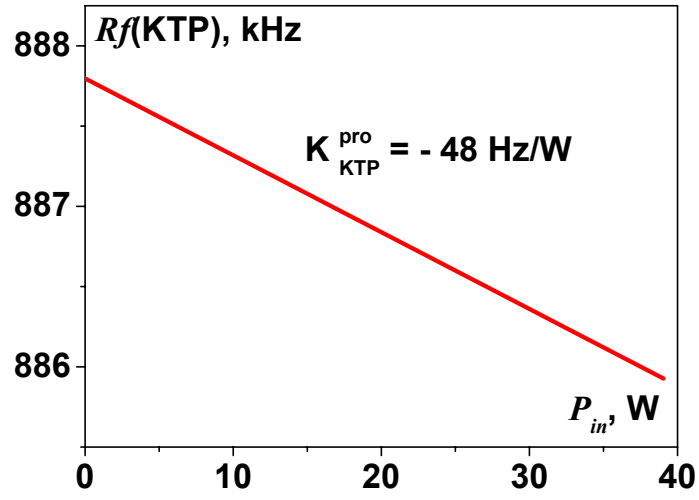

FIG. 8 Piezoelectric resonance frequency shift with the incident laser power measured for KTP crystal.

It was observed that the change in the resonance line shape measured in the experiment with uniform ambient temperature change and under non-uniform laser heating were identical. Basing on this fact we introduce a novel notion of the equivalent heating temperature of the crystal, which can characterize its non-uniform heating caused by laser radiation.

The dependence of the resonance frequency on laser power $R f_{n}\left(P_{\text {in }}\right)$ is determined primarily by the crystal unknown optical absorption coefficient $\alpha(\lambda)$ ( $\lambda$ is the radiation wavelength) and heat exchange conditions at the crystal-air boundary. At the wavelength $\lambda=1064 \mathrm{~nm}$ the KTP crystal exhibits linear dependence of $R f_{n}\left(P_{\text {in }}\right)$ (see Figure 8) because of the weak linear absorption and negligible nonlinear absorption in the used power range (up to $70 \mathrm{~W}$ ). It enables one to express the equivalent crystal heating temperature $\Delta \Theta^{\mathrm{eq}}\left(P_{i n}\right)$ in terms of the piezoelectric thermal $K_{n}^{p r t}$ and optical $K_{n}^{\text {pro }}$ coefficients of some resonance mode:

$$
\Delta \Theta^{\mathrm{eq}}\left(P_{i n}\right)=\frac{\Delta R f_{n}\left(P_{i n}\right)}{K_{n}^{p r t}}=\frac{K_{n}^{p r o}}{K_{n}^{p r t}} P_{i n}
$$

The equivalent crystal temperature can then be written as

$$
\Theta_{c r}^{\mathrm{eq}}\left(P_{i n}\right)=\theta_{a}+\Delta \Theta_{c r}^{\mathrm{eq}}\left(P_{i n}\right)
$$

Dependence of the equivalent crystal temperature on the KTP incident laser power measured for the KTP, QT1 and QT2 crystals is shown in Figure 11. 


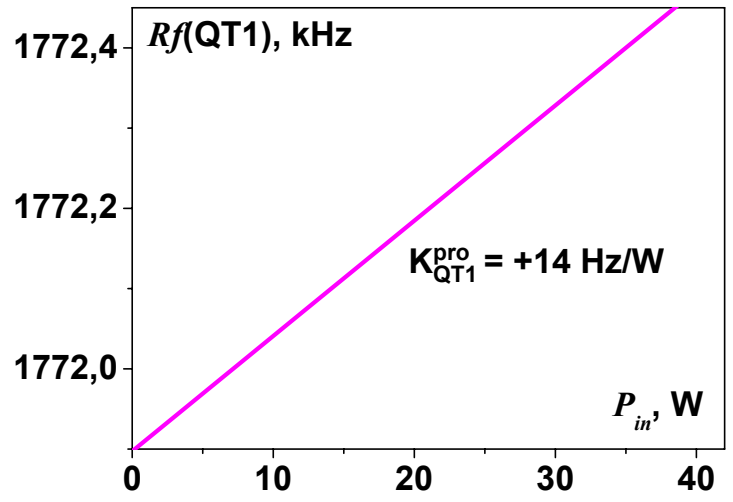

FIC. 9 Dependence of the QT1 piezoelectric resonance frequency on the KTP incident laser power.

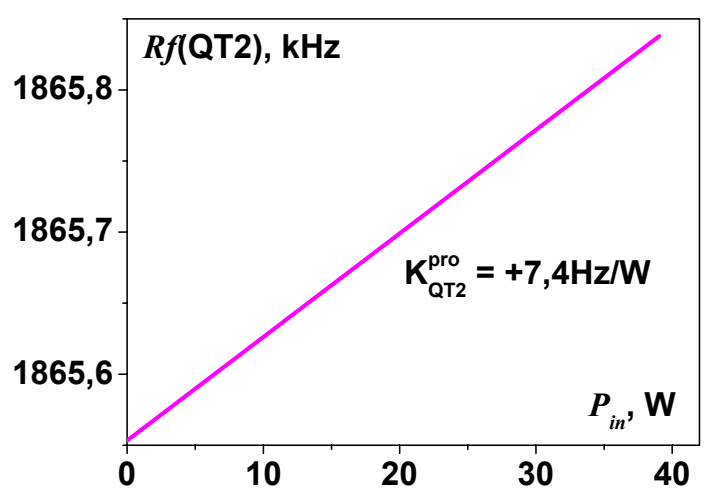

FIG. 10 Dependence of the QT2 piezoelectric resonance frequency on the KTP incident laser power.

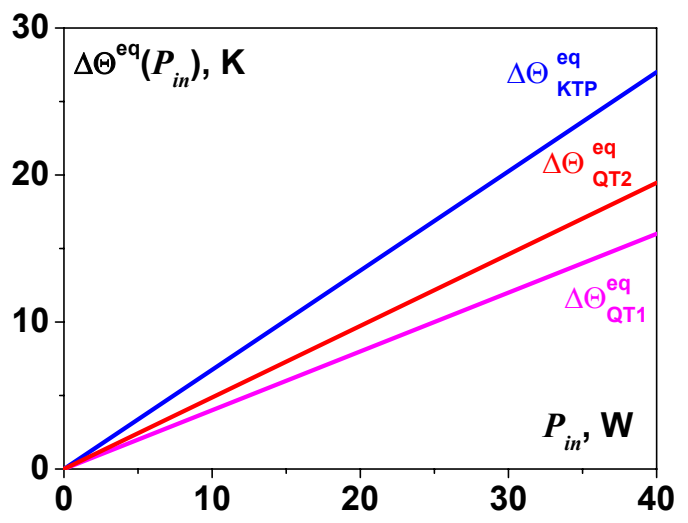

FIG. 11 Equivalent heating temperature dependence on the KTP incident laser power measured for the KTP, QT1 and QT2 crystals.

True spatial thermodynamic temperature distribution inside the crystal $\theta_{c r}\left(x, y, z, P_{i n}\right)$ can be expressed as

$$
\theta_{c r}\left(x, y, z, P_{i n}\right)=\theta_{a}+\Delta \Theta_{c r}^{\mathrm{eq}}\left(P_{\text {in }}\right)+\delta \theta\left(x, y, z, P_{\text {in }}\right)
$$

Our numerical model shows that for the majority of the experimental situations

$$
\delta \theta\left(x, y, z, P_{\text {in }}\right)<<\Delta \Theta_{c r}^{\mathrm{eq}}\left(P_{\text {in }}\right)
$$

\section{CALCULATIONS}

\subsection{Heating model}

The temperature distribution inside the crystal is governed by a heat conduction equation. We assume that the convective heat transfer takes place at the crystal air interface. When crystal heating exceeds $30-40 \mathrm{~K}$ the thermal radiation should be taken into account.

The crystal is assumed to be long enough to neglect thermal effects on the input and output facets as well as temperature $\mathrm{z}$-dependence. In this case the heat conduction problem could be written as:

$$
\begin{array}{ll}
\rho_{c r} c_{c r} \frac{\partial \theta}{\partial t}=\kappa_{c r}\left(\frac{\partial^{2} \theta}{\partial x^{2}}+\frac{\partial^{2} \theta}{\partial y^{2}}\right)+\alpha I & \text { in crystal } \\
\rho_{a} c_{a} \frac{\partial \theta}{\partial t}=\kappa_{a}\left(\frac{\partial^{2} \theta}{\partial x^{2}}+\frac{\partial^{2} \theta}{\partial y^{2}}\right) & \text { in air } \\
-\kappa_{a} \frac{\partial \theta}{\partial n}=h^{T}\left(\theta_{c r}-\theta_{a}\right) & \text { at crystal-air boundary } \\
\theta=\theta_{0} & \text { at outer boundary. }
\end{array}
$$

Here indices ' $c r$ ', ' $a$ ' are related to the crystal and air, respectively, $t$ is time, $n$ is a crystal-air boundary normal vector; $c_{c r}, c_{a}$ are specific heats, $\rho_{c r}, \rho_{a}-$ densities; $\kappa_{c r}, \kappa_{a}-$ thermal conductivities; $h^{T}-$ unknown heat transfer coefficient at the crystal-air interface. The outer boundary is generally chosen on the electrodes. The calculation domain is a rectangle $l_{X} \times l_{Y}, l_{X}(9 \mathrm{~mm})>L_{x}, l_{Y}(15 \mathrm{~mm})>L_{y}$, where $L_{x}(3.2 \mathrm{~mm}), L_{y}(2.8 \mathrm{~mm})$ are the crystal transverse dimensions. It is assumed also that the laser radiation is transmitted only through the crystal. In our experiments we used a singlemode laser, so the intensity distribution was close to Gaussian.

$$
I(x, y)=\frac{P_{\text {in }}}{2 \pi w^{2}} \exp \left(-\frac{x^{2}+y^{2}}{2 w^{2}}\right)
$$

where $w$ is a beam radius at $1 / e^{2}$ level. We considered only a stationary solution of the problem. The values of $h^{T}, \alpha$ are fitted to obtain the experimentally measured temperature of the sample crystal and the air temperature at the positions of the small quartz crystals. The system (9) is solved by a finitedifference method [13]. A uniform grid of $N_{x} \times N_{y}$ points on the $l_{X} \times l_{Y}$ area is introduced:

$$
\begin{array}{lll}
x_{i, j}=i h_{x}-L_{X} / 2, & i=0 \ldots N_{x}, & h_{x}=L_{X} / N_{x} \\
y_{i, j}=j h_{y}-L_{Y} / 2, & j=0 \ldots N_{y}, & h_{y}=L_{Y} / N_{y}
\end{array}
$$

The temperature values in the grid nodes are numbered by two indices $i, j$. The second derivatives are approximated as follows:

$$
\begin{aligned}
& \left(\frac{\partial^{2} \theta}{\partial x^{2}}\right)_{i, j} \approx \frac{\theta_{i+1, j}-2 \theta_{i, j}+\theta_{i-1, j}}{h_{x}^{2}} \\
& \left(\frac{\partial^{2} \theta}{\partial y^{2}}\right)_{i, j} \approx \frac{\theta_{i, j+1}-2 \theta_{i, j}+\theta_{i, j-1}}{h_{y}^{2}}
\end{aligned}
$$

For the crystal and air interior points we obtain the following equations:

$$
\begin{aligned}
& \frac{1}{h_{x}^{2}} \theta_{i+1, j}+\frac{1}{h_{x}^{2}} \theta_{i-1, j}+\frac{1}{h_{y}^{2}} \theta_{i, j+1}+\frac{1}{h_{y}^{2}} \theta_{i, j-1}-2 \theta_{i, j}\left(\frac{1}{h_{x}^{2}}+\frac{1}{h_{y}^{2}}\right) \\
& =-\frac{\gamma \alpha P_{i n}}{2 \pi \kappa_{c r} w^{2}} \exp \left(-\frac{x_{i, j}^{2}+y_{i, j}^{2}}{2 w^{2}}\right),
\end{aligned}
$$




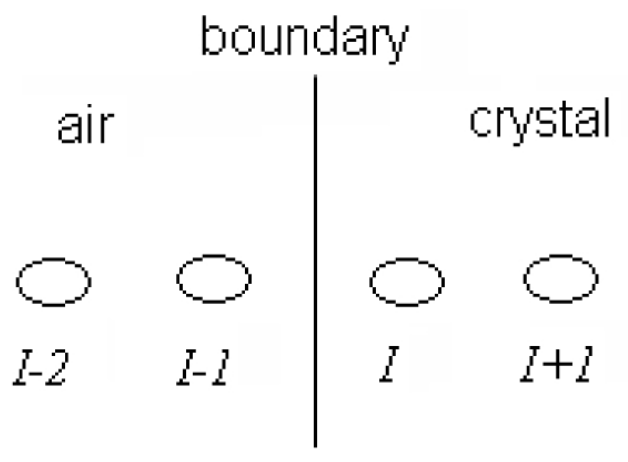

FIG. 12 Convective boundary condition for the left boundary of the KTP crystal with the surrounding air.

where $\gamma=1$ for the points inside the sample crystal, and $\gamma=0$ for the points inside the air region.

In what follows we obtain a finite-difference equation for a convective boundary condition. The effect of the thermal resonators QT1, QT2 on the convective heat exchange condition is neglected. Let's consider a vertical boundary where air is located to the left of the boundary, and crystal is on the right side (Figure 12). Let's assume that the boundary is located between nodes $(I-1, J)$ and $(I, J)$. For nodes $(I-2, J)$ and $(I+1, J)$ the equations in the form (12) can be written while for nodes $(I$ $-1, J)$ and $(I, J)$ this cannot be done because of medium discontinuity. In that case for node $(I, J)$ we use the following equations obtained from (9):

$$
\kappa_{\mathcal{C}} \frac{\theta_{I+1, j}-\theta_{I, j}}{h_{x}}=h^{T}\left(\theta_{I, j}-\theta_{I-1, j}\right)
$$

And for the node $(I-1, J)$ we use:

$$
\kappa_{a} \frac{\theta_{I-1, j}-\theta_{I-2, j}}{h_{x}}=h^{T}\left(\theta_{I, j}-\theta_{I-1, j}\right)
$$

In a similar way the equations for the other boundary parts can be written. We assume that the thickness of the aircrystal interface layer is less than the step of the selected grid. Eqs. (13)-(15) constitute linear algebra problem that can be solved using standard methods.

\subsection{Modeling results for KTP crystal}

We used the model described above for the following experimental parameters. The laser source was a single-mode linearly polarized laser with a radiation power of $35 \mathrm{~W}$ and a laser beam diameter of $1.5 \mathrm{~mm}$ (FWHM). The distances between the KTP and the QT crystals facets were approximately $0.5 \mathrm{~mm}$ each. The KTP crystal transverse dimensions were $3.2 \times 2.8 \mathrm{~mm}$, the crystal length was $12 \mathrm{~mm}$. We used the following material constants for KTP: $\rho_{c r}=3 \cdot 10^{3} \mathrm{~kg} / \mathrm{m}^{3}, c_{c r}=730 \mathrm{~J} /(\mathrm{kg} \mathrm{K}), \kappa_{c r}=3 \mathrm{~W} /(\mathrm{m} \mathrm{K})$, and for air (at normal conditions):

$$
\rho_{a}=1.29 \mathrm{~kg} / \mathrm{m}^{3} c_{a}=1000 \mathrm{~J} /(\mathrm{kg} \mathrm{K}), \kappa_{a}=0.026 \mathrm{~W} /(\mathrm{m} \mathrm{K}) .
$$

The mathematical modeling resulted in the KTP equivalent heating temperature $\Delta \Theta^{e q}(\mathrm{QT} 1)=25 \mathrm{~K}$ and thermal resonators equivalent heating temperatures $\Delta \Theta^{e q}(\mathrm{KTP})=17 \mathrm{~K}$,

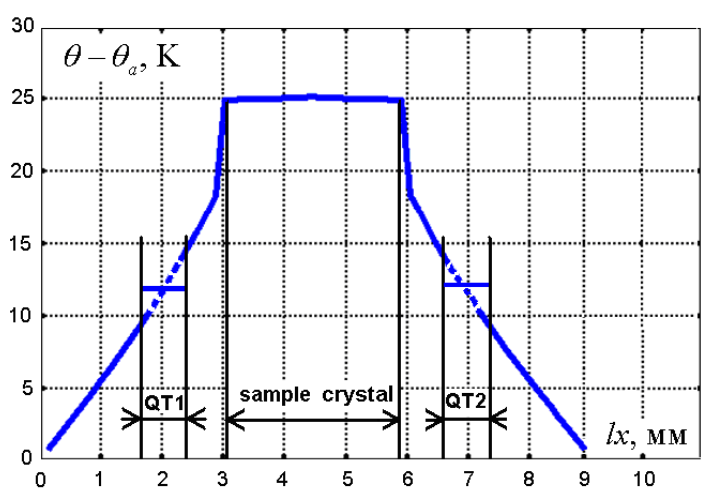

FIG. 13 Temperature distribution along the $x$-axis calculated for the KTP crystal and surrounding air for the incident laser power $35 \mathrm{~W}$.

$\Delta \Theta^{e q}(\mathrm{QT} 2)=14 \mathrm{~K}$. The calculated spatial non-uniform temperature distribution is shown in Figure 13.

We also obtained the following KTP crystal parameters from fitting the experimental data using our model: optical absorption coefficient $\alpha(\lambda=1.06 \mu \mathrm{m})=0.7 \cdot 10^{-3} \mathrm{~cm}^{-1}$, heat transfer coefficient $h^{T}=30 \mathrm{~W} /\left(\mathrm{m}^{2} \mathrm{~K}\right)$.

It is clearly seen from Figure 13 that the temperature gradient inside the crystal is extremely small (less than $0.01 \mathrm{~K} / \mathrm{mm}$ ) while the temperature gradient in vicinity of the sample crystal external surface is relatively high $(6 \mathrm{~K} / \mathrm{mm})$. Therefore the conventional methods of the crystal temperature measurement give inevitably high errors. Temperature measured at the QT1 and QT2 resonators positions could serve as an example of this assertion: the distance uncertainty for the QT1 and QT2 resonators positions with respect to the sample crystal is $\approx 0.1 \mathrm{~mm}$, while their measured temperature difference is as high as $\approx 3 \mathrm{~K}$.

\section{CONCLUSIONS}

We have demonstrated that nonlinear-optical crystal temperature during laser radiation interaction can be precisely measured from the crystal piezoelectric resonance frequency shift. This gives a pathway to better control of frequency conversion in the high-power applications. A novel notion in laser physics, "the equivalent temperature" of the non-uniformly heated crystal has been introduced. It gives a possibility for a substitution of the true thermodynamic crystal temperature function, which cannot be measured directly and can be only calculated by making several assumptions, by the experimentally measurable scalar parameter, the equivalent temperature. We have also demonstrated a method for the optical absorption and heat transfer coefficients measurement for nonlinear-optical crystals. It is based on stationary measurements of the equivalent temperature of the laser heated nonlinear-optical crystal and the collocated quartz thermal resonators and fitting of the experimental data to the appropriate heating model. The method is applicable to the laser powers varied by 5-6 orders of magnitude from $\mathrm{mW}$ to $\mathrm{kW}$ level. 


\section{AKNOLEDGEMENTS}

We are grateful to Dr. D.A. Oulianov (NTO "IRE-Polus", Russia) for helpful discussions.

\section{References}

[1] V. G. Dmitriev, and L. V. Tarasov Applied Nonlinear Optics (Phyzmatlit, Moscow, 2004) in Russian.

[2] B. Boulanger, I. Rousseau, J. P. F'eve et al "Optical studies of laserinduced gray-tracking in KTP" IEEE Journal of Quantum Electronics 35, No.3, 281-286 (1999).

[3] M. N. Satyanarayan, and H. L. Bhat "Electrical and optical characterization of electrochromic damages in KTP single crystals" Journal of the Korean Physical Society 32, 420-423 (1998).

[4] A. Hildenbrand, F. R. Wagner, H. Akhouayri et al "Laser-induced damage investigation at $1064 \mathrm{~nm}$ in $\mathrm{KTiOPO} 4$ crystals and its analogy with RbTiOP04" Applied Optics 48, No. 21, 4263-4269 (2009).

[5] X. Mu, and Y. J. Ding "Investigation of damage mechanisms of KTiOPO 4 crystals by use of a continuous-wave argon laser" Applied Optics 39, No. 18, 3099-3103 (2000).

[6] U. Willamowsky, D. Ristau, and E. Welsch "Measuring the absolute absorptance of optical laser components" Applied Optics 37, No. 36, 8362-8370 (1998).

[7] 0. A. Ryabushkin, A. V. Konyashkin, and D. V. Myasnikov "Equivalent temperature of crystal interacting with laser radiation" in Proceeding of the EOS Annual Meeting 2010 (EOS, Paris, 2010).
[8] A. V. Konyashkin, A. V. Doronkin, V. A. Tyrtyshnyy, and 0. A. Ryabushkin "A Radio-Frequency Impedance Spectroscope for Studying Interaction of High-Power Laser Radiation with Crystals" Instruments and Experimental Techniques 52, No. 6, 816-823 (2009).

[9] C. Yu, M. J. McKenna, J. D. White, and J. D. Maynard "A new resonant photoacoustic technique for measuring very low optical absorption in crystals and glasses" J. Acoustical Society of America 91, No. 2, 868-877 (1992).

[10] F. Bezancon, J. Mangin, P. Stimer, and M. Maglione "Accurate determination of the weak optical absorption of piezoelectric crystals used as capacitive massive bolometers" IEEE Journal of Quantum Electronics 37, No 11, 1396-1400 (2001).

[11] V. A. Tyrtyshnyy, A. V. Konyashkin, and 0. A. Ryabushkin "Piezoelectric resonator for optical power measurement with radiation beam quality conservation" Instruments and Experimental Techniques 54, No. 2 (2011) (to be published).

[12] D. Myasnikov, A. Konyashkin, and 0. Ryabushkin "Identification of eigenmodes of volume piezoelectric resonators in resonant ultrasound spectroscopy" Technical Physics Letters 36, No. 7, 632-635 (2010).

[13] K. W. Morton, and D. E. Mayers Numerical solutions of partial differential equations (Cambridge University Press, Cambridge, 2005). 